\title{
Correspondence
}

\section{Prevalence of antidepressant-induced sexual dysfunction among psychiatric outpatients attending a tertiary care hospital}

\section{To the Editor}

I have read with interest the original article entitled "Prevalence of antidepressant-induced sexual dysfunction among psychiatric outpatients attending a tertiary care hospital" by Mohammed AlBreiki et al, published in Neurosciences journal. ${ }^{1}$ I want to commend the authors for selecting an interesting topic with a wide range of age, absence of organic diseases that causes sexual dysfunction, and the patients used 4 different antidepressant compounds. There are certain methodological concerns which might create a response bias among patients suffering from sexual dysfunction.

First, the statistical analysis; the authors described in the section Data Management, they described the data with Confidence Interval (CI), and they used analysis of variance (ANOVA) test, but they are not available in the results.

Second, the authors reported premature ejaculation in $55.6 \%$ of male participants. In fact, fluoxetine and paroxetine $(20 \mathrm{mg})$ is effective in the management of premature ejaculation. ${ }^{2,3}$ Moreover, the authors reported 32 patients sexual dysfunction were treated with mirtazapine, while mirtazapine does not cause sexual dysfunction as other selective serotonin reuptake inhibitors (SSRIs). ${ }^{4}$ In addition, venlafaxine, a norepinephrine-serotonin reuptake inhibitor, was reported as an effective drug in the management of premature ejaculation. ${ }^{5}$ The SSRIs are useful in management sexual dysfunction in men, and unlikely to cause erectile failure.

Third, SSRIs are most likely to cause sexual dysfunction in women, and unlikely to show broad spectrum of sexual dysfunction in men. ${ }^{6}$

To my knowledge, this cross-sectional study is of great importance because elements of originality are present. The following are the recommendations for future researches: First, determination the scores of depression and anxiety scales for each sexual dysfunction to exclude the cause of sexual dysfunction is related to the psychiatric illness and not to SSRI, particularly in men. Second, proper statistical analysis definitely will improve the quality of any study.

\section{Marwan S. Al-Nimer, Department of Pharmacology and Toxicology, College of Pharmacy, Hawler Medical University Kurdistan Region, Iraq}

\section{Reply from the Author}

Firstly, I feel proud that this paper resonated such echo among eminent researchers and pharmacological companies from around the world. I received many productive comments and requests for collaboration from drug companies to discuss out results further. This reflects not only the importance of this paper but the impact of the neurosciences journal and its global respect and credit to the published papers and the precious comments of the reviewers.

Secondly, I would like to thank Dr. Marwan for his interest in the paper and his comments. I agree with Dr. Marwan, may be if we investigated the results deeper with ANOVA and used CI when appropriate, surely our findings will be more statistically powerful. We avoided this in the paper to prevent excessive details. We used Chi square test and $p$-value to elucidate the significance of the differences between variables.

With regard to the finding of premature ejaculation caused by drugs that actually used to treat premature ejaculation, namely paroxetine, fluoxetine and venlafaxine. We noticed this finding during analysis and elicited long discussion between the authors. This is an original study thus honest report of the finding is a must. I may summarize my explanation in 3 points:

First, the pharmacodynamics and the pharmacokinetic may differ in the population under study. Thus, SSRIs are not always effective in treating premature ejaculation. Second, the report of premature and delayed ejaculation is subjective, thus this finding may not be reported a such under standardized parameters. Third, Many medications have this phenomenon of paradox side effects such as benzodiazepine paradox anxiety and irritability. ${ }^{7}$

With regard to the gender differences in sexual dysfunction caused by antidepressants, males are more likely to suffer from erectile dysfunction and females are more likely to suffer from anorgasmia. However, the results of studies are not consistent. ${ }^{8}$

Again, I healthfully thank Dr. Al-Nimer for his comments and feedback. The recommendations will be surely considered for future researches. I especially liked the idea of correlating the depression and anxiety scores with the onset of antidepressants induced sexual dysfunctions.

\section{Mandhar Al-Maqbali, Department of Psychiatry, Sohar Hospital, Sohar, Oman.}

\section{References}

1. AlBreiki M, AlMaqbali M, AlRisi K, AlSinawi H, Al Balushi M, Al Zakwani W. Prevalence of Antidepressant-Induced Sexual Dysfunction Among Psychiatric Outpatients Attending a Tertiary Care Hospital. Neurosciences (Riyadh) 2020; 25: 55-60. 
2. Siroosbakht S, Rezakhaniha S, Rezakhaniha B. Which of available selective serotonin reuptake inhibitors (SSRIs) is more effective in treatment of premature ejaculation? A randomized clinical trial. Int Braz J Urol 2019; 45: 1209-1215.

3. Balci M, Atan A, Senel C, Guzel O, Aslan Y, Lokman U, et al. Comparison of the treatment efficacies of paroxetine, fluoxetine and dapoxetine in low socioeconomic status patients with lifelong premature ejaculation. Cent European J Urol 2019; 72: 185-190.

4. Clayton AH, Croft HA, Handiwala L. Antidepressants and sexual dysfunction: mechanisms and clinical implications. Postgrad Med 2014; 126: 91-99.

5. Safarinejad MR. Safety and efficacy of venlafaxine in the treatment of premature ejaculation: A double-blind, placebocontrolled, fixed-dose, randomised study. Andrologia 2008; 40: $49-55$.
6. Rappek NAM, Sidi H, Kumar J, Kamarazaman S, Das S, Masiran R, et al. Serotonin selective reuptake inhibitors (SSRIs) and female sexual dysfunction (FSD): Hypothesis on its association and options of treatment. Curr Drug Targets 2018; 19: $1352-1358$.

7. Camkurt MA. Spontaneous ejaculation; caused by venlafaxine, reverted by mirtazapine. The European Research Journal 2015; 1: 157-159.

8. Kennedy SH, Eisfeld BS, Dickens SE, Bacchiochi JR, Bagby RM. Antidepressant-induced sexual dysfunction during treatment with moclobemide, paroxetine, sertraline, and venlafaxine. J Clin Psychiatry 2000; 61: 276-281. 